\title{
Synthesis, characterization and antibacterial evaluation of new pyridyl-thiazole hybrids of sulfonamides
}

\author{
Zafer Șahin' (1), Sevde Nur Biltekin² (D), Leyla Yurttaș³ (D), Șeref Demirayak (1) \\ 'Istanbul Medipol University, School of Pharmacy, Department of Pharmaceutical Chemistry, İstanbul, Turkey \\ 2Istanbul Medipol University, School of Pharmacy, Department of Pharmaceutical Microbiology, İstanbul, Turkey \\ ${ }^{3}$ Anadolu University, Faculty of Pharmacy, Department of Pharmaceutical Chemistry, Eskișehir, Turkey
}

ORCID IDs of the authors: Z.Ș. 0000-0002-5976-676X; S.N.B. 0000-0003-1896-2729; L.Y. 0000-0002-0957-6044; S.D. 0000-0002-0841-1299

Cite this article as: Sahin, Z., Biltekin, S.N., Yurttas, L., \& Demirayak, S. (2021). Synthesis, characterization and antibacterial evaluation of new pyridyl-thiazole hybrids of sulfonamides. İstanbul Journal of Pharmacy, 51 (1), 67-72.

\begin{abstract}
Background and Aims: Sulfonamide drugs are a very old and noted group of small molecules, and are still one of the most important antimicrobial compounds. In this study, starting from sulfonamide drugs, new original compounds containing frequent and functional rings such as thiazole and pyridine were synthesized and their antimicrobial effects were evaluated. Methods: Eighteen new compounds were synthesized by converting the 4-amino group of the sulfonamides to thiourea, and continued by thiazole ring closure. Characterization of the compounds was carried out by FT-IR, ${ }^{1} \mathrm{H}-\mathrm{NMR}$ and ${ }^{13} \mathrm{C}-\mathrm{NMR}$ and HRMS. MIC values were obtained in antimicrobial activity studies, which were carried out by Broth Microdilution method. Results: Compounds $\mathbf{3 p - r}$ had an effect of $32 \mu \mathrm{g} / \mathrm{ml}$ against $B$. spizizenii. In addition, compounds $\mathbf{3 d}-\mathbf{f}$ and $\mathbf{3 p - r}$ each showed effect against different gram-positive bacteria. Compound $3 \mathrm{r}$ had an MIC of $128 \mu \mathrm{g} / \mathrm{mL}$ against gram-negative organisms. The rest of the series did not affect gram-negative bacteria. In the study, chloramphenicol and sulfamethoxazole were used as standards.

Conclusion: Sulfanilamide and sulfadiazine derivatives showed higher inhibitory effects compared to the rest of the series. $\mathbf{3 d - f}$ and $\mathbf{3 p - r}$ showed inhibitor activity against gram-positive bacteria, conversely to the standard drug sulfamethoxazole, which possibly means that the mechanism of action is not same.
\end{abstract}

Keywords: Sulfonamide, thiazole, antibacterial

\section{INTRODUCTION}

Infectious diseases have been an incessant problem for human over the years. Gram positive and gram negative bacteria cause diverse infectious reactions. Among the microbial strains, E. coli produce lethal toxins, which can be contaminated from unwashed foods (Donnenberg \& Whittam, 2001). P. aeruginosa and K. pneumoniae cause respiratory diseases (Gellatly \& Hancock, 2013; Bengoechea \& Pessoa 2018; Russo \& Marr 2019). B. spizizenii is a subspecies of the Bacillus family and may produce typical Bacillus infections (Drobniewski, 1993). S. aureus, E. faecalis, S. epidermidis and vancomycin-resistant enterococcus (VRE) are other pathogenic microorganisms involved in this study. Many drug classes and targets are identified for treatment of these diseases. One of the most important classes among them is the sulfonamides, with many drugs identified; sulfamethoxazole, sulfapyridine, sulfadiazine etc. (Supuran, 2017). Advances in the development of new drugs are dependent on synthesis of original molecules and evaluation of their activity on different microbial strains. Pyridine and thiazole are frequent rings used in approved and investigational drugs because they provide ionizable groups and/or good pharmacokinetical properties. Besides, pyridyle- 
thiazole analogs are an investigational pharmacophore against different targets. The feature for pyridyle-thiazole analogs is their potential for complex formation of cofactors as they are bearing close nitrogen atoms on related rings. (Kashyap et al., 2011; Hamada, 2018; Ertas et al., 2018) Similar sulfonamide derivatives have been reported in a variety of pharmacological activities such as sodium channel inhibitors, anticancer or anti-inflammatory properties in the literature (Sun et al. 2014; El-Sayed et al., 2010). In this study, we synthesized 18 novel pyridyle-thiazole sulfonamide hybrids to evaluate their antimicrobial properties.

\section{MATERIALS AND METHODS}

\section{Chemistry}

The reactants necessary for the synthesis process were purchased from Sigma Aldrich Chemical Corp. Melting point of title molecules were accomplished by a Stuart melting point apparatus and experiments performed in duplicate. Infra-red spectrums were recorded by Perkin Elmer Spectrom Two using attenuated total reflection (ATR) method. ${ }^{1} \mathrm{H}-\mathrm{NMR}$ and ${ }^{13} \mathrm{C}-\mathrm{NMR}$ spectrums were recorded in Bruker $300 \mathrm{MHz}$ UltraShield NMR and Bruker $75 \mathrm{MHz}$ UltraShield NMR, respectively. DMSO- $d_{6}$ was used as solvent and TMS was used as standard. High-resolution mass spectrums were recorded in Shimadzu 8,040 LC/MS/MS ITTOF system by the electron spray method (ESI).

\section{Synthesis of sulfonamide-thioureas (1a-f)}

Related sulfonamide drug (100 mmol), equal mole (100 mmol) ammonium thiocyanate was dissolved in distilled water by $\mathrm{HCl}$ addition (Figure 1). The mixture was refluxed for 8 hours and then left to reach room temperature. The precipitation was collected and recrystallized from ethanol.

\section{Synthesis of bromoacetylpyridines (2a-c)}

Acetyl pyridine derivatives were dissolved in hydrobromic acid solution (48\%) and bromine added drop wise. The reaction mixture was stirred for 1 hour and the precipitation collected. It was crystallized from ethanol.

\section{Synthesis of $\mathbf{N}$-(pyridyle)thiazolyl sulfonamides (3a-r)}

Equal moles (10 mmol) of bromoacetyl pyridine (2a-c) and sulfonamide thiourea (1a-f) derivatives were dissolved in ethanol and refluxed for 4 hours. The precipitated product was filtered, poured into water at room temperature and neutralized by sodium acetate. If precipitation did not occur, water was added to provide precipitation. The products were recrystallized from ethanol.

\section{N-(4,6-dimethylpyrimidin-2-yl)-4-((4-(pyridine-2-yl)thi- azol-2-yl)amino)benzene-sulfonamide (3a)}

Yield: 77\%, m.p. $253^{\circ} \mathrm{C}$, IR v̄max $\left(\mathrm{cm}^{-1}\right)$ : $3351.25(\mathrm{~N}-\mathrm{H}), 3055.31$ (N-H), 3005.95, 2927.74 (Ar C-H). ${ }^{1} \mathrm{H}-\mathrm{NMR}\left(300 \mathrm{MHz}, \mathrm{DMSO}-d_{6}\right.$

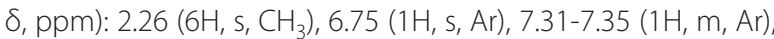
$7.65(1 \mathrm{H}, \mathrm{s}, \mathrm{Ar}), 7.84-7.92(3 \mathrm{H}, \mathrm{m}, \mathrm{Ar}), 7.97(2 \mathrm{H}, \mathrm{d}, \mathrm{J}: 8.89 \mathrm{~Hz}, \mathrm{Ar})$ $8.06(2 \mathrm{H}, \mathrm{d}, \mathrm{J}: 7.93 \mathrm{~Hz}, \mathrm{Ar}), 8.57-8.61(1 \mathrm{H}, \mathrm{m}, \mathrm{Ar}), 10.75(1 \mathrm{H}, \mathrm{s}, \mathrm{NH})$ $11.46(1 \mathrm{H}, \mathrm{s}, \mathrm{NH}) .{ }^{13} \mathrm{C}-\mathrm{NMR}\left(75 \mathrm{MHz}, \mathrm{DMSO}-d_{6}, \delta, \mathrm{ppm}\right): 23.45$, 108.63, 116.15, 121.05, 123.27, 130.22, 132.45, 137.83, 144.92, 149.89, 150.87, 152.36, 156.83, 162.97 HR-MS (M+H): pred: 439.1005, found: 439.1002 .<smiles>[R]Cc1csc(Nc2ccc(S(=O)(=O)N[Y])cc2)n1</smiles>

\begin{tabular}{|l|l|l|l|l|l|}
\hline $\mathbf{C}$ & $\mathbf{R 1}$ & $\mathbf{R 2}$ & $\mathbf{C}$ & $\mathbf{R 1}$ & $\mathbf{R 2}$ \\
\hline $\mathbf{3 a}$ & 3,5-Dimethylpyrimidine-2-yl & 2-Pyridyle & $\mathbf{3 j}$ & 5-methylizoxazole-3-yl & 2-Pyridyle \\
\hline $\mathbf{3 b}$ & 3,5-Dimethylpyrimidine-2-yl & 3-Pyridyle & $\mathbf{3 k}$ & 5-methylizoxazole-3-yl & 3-Pyridyle \\
\hline $\mathbf{3 c}$ & 3,5-Dimethylpyrimidine-2-yl & 4-Pyridyle & $\mathbf{3 l}$ & 5-methylizoxazole-3-yl & 4-Pyridyle \\
\hline $\mathbf{3 d}$ & Pyrimidine-2-yl & 2-Pyridyle & $\mathbf{3 m}$ & Thiazole-2-yl & 2-Pyridyle \\
\hline $\mathbf{3 e}$ & Pyrimidine-2-yl & 3-Pyridyle & $\mathbf{3 n}$ & Thiazole-2-yl & 3-Pyridyle \\
\hline $\mathbf{3 f}$ & Pyrimidine-2-yl & 4-Pyridyle & $\mathbf{3 0}$ & Thiazole-2-yl & 4-Pridyle \\
\hline $\mathbf{3 g}$ & Pyridine-2-yl & 2-Pyridyle & $\mathbf{3 p}$ & H & 2-Pyridyle \\
\hline $\mathbf{3 h}$ & Pyridine-2-yl & 3-Pyridyle & $\mathbf{3 q}$ & H & 3-Pyridyle \\
\hline $\mathbf{3 i}$ & Pyridine-2-yl & 4-Pyridyle & $\mathbf{3 r}$ & H & 4-Pyridyle \\
\hline
\end{tabular}

Figure 1. Synthesis of compounds 3a-r. 
N-(4,6-dimethylpyrimidin-2-yl)-4-((4-(pyridine-3-yl)thiazol-2-yl)amino)benzene-sulfonamide (3b)

Yield: 66\%, m.p. $265^{\circ} \mathrm{C}$, IR v̄max $\left(\mathrm{cm}^{-1}\right)$ : $3052.88(\mathrm{~N}-\mathrm{H}), 2925.56$ (Ar C-H). ${ }^{1} \mathrm{H}-\mathrm{NMR}(300 \mathrm{MHz}$, DMSO-d 6 , $\delta, \mathrm{ppm}): 2.25\left(6 \mathrm{H}, \mathrm{s}, \mathrm{CH}_{3}\right)$, $6.75(1 \mathrm{H}, \mathrm{s}, \mathrm{Ar}), 7.47-7.55(1 \mathrm{H}, \mathrm{m}, \mathrm{Ar}), 7.65(1 \mathrm{H}, \mathrm{s}, \mathrm{Ar}), 7.85(2 \mathrm{H}$, d, J: $8.62 \mathrm{~Hz}, \mathrm{Ar}), 7.98(2 \mathrm{H}, \mathrm{d}, \mathrm{J}: 8.93 \mathrm{~Hz}, \mathrm{Ar}), 8.29(1 \mathrm{H}, \mathrm{d}, \mathrm{J}: 8.07$ $\mathrm{Hz}, \mathrm{Ar}), 8.53(1 \mathrm{H}$, br. s, Ar), $9.17(1 \mathrm{H}, \mathrm{br} . \mathrm{s}, \mathrm{Ar}), 10.78(1 \mathrm{H}, \mathrm{s}, \mathrm{NH})$, $11.51(1 \mathrm{H}, \mathrm{s}, \mathrm{NH}) .{ }^{13} \mathrm{C}-\mathrm{NMR}\left(75 \mathrm{MHz}\right.$, DMSO- $\left.d_{6} \delta, \mathrm{ppm}\right): 23.44$, $106.43,114.12,116.19,124.27,130.25,132.51,133.41,144.84$, 147.50, 147.81, 149.03, 156.81, 163.20, 167.82. HR-MS (M+H): pred: 439.1005 , found: 439.1002

N-(4,6-dimethylpyrimidin-2-yl)-4-((4-(pyridine-4-yl)thiazol-2-yl)amino)benzene-sulfonamide (3c)

Yield: 60\%, m.p. $266^{\circ} \mathrm{C}$, IR ūmax $\left(\mathrm{cm}^{-1}\right)$ : $3264.27(\mathrm{NH}), 3185.09(\mathrm{~N}$ H), 3103.63 (Ar C-H). ${ }^{1} \mathrm{H}-\mathrm{NMR}\left(300 \mathrm{MHz}\right.$, DMSO- $\left.d_{6}, \delta, \mathrm{ppm}\right): 2.26$ $\left(6 \mathrm{H}, \mathrm{s}, \mathrm{CH}_{3}\right), 6.76(1 \mathrm{H}, \mathrm{s}, \mathrm{Ar}), 7.87(2 \mathrm{H}, \mathrm{d}, \mathrm{J}: 8.97 \mathrm{~Hz}, \mathrm{Ar}), 7.98(2 \mathrm{H}, \mathrm{d}, \mathrm{J}$ : $8.94 \mathrm{~Hz}, \mathrm{Ar}), 8.17-8.29$ (3H, m, Ar), 8.79-???? (2H, m, Ar), $10.93(1 \mathrm{H}$, $\mathrm{s}, \mathrm{NH}), 11.55(1 \mathrm{H}, \mathrm{s}, \mathrm{NH}) .{ }^{13} \mathrm{C}-\mathrm{NMR}\left(75 \mathrm{MHz}, \mathrm{DMSO}-d_{6}, \delta, \mathrm{ppm}\right)$ : 23.44, 114.12, 116.44, 121.96, 130.21, 132.91, 144.52, 145.93, 156.79, 163.46. HR-MS (M+H): pred: 439.1005, found: 439.1001.

4-((4-(pyridine-2-yl)thiazol-2-yl)amino)-N-(pyrimidin-2yl)benzenesulfonamide (3d)

Yield: 78\%, m.p. $226^{\circ} \mathrm{C}$, IR v̄max $\left(\mathrm{cm}^{-1}\right)$ : $3282.96(\mathrm{NH}), 3194.78$ (N-H), 3083.00-2943.44 (Ar C-H). ${ }^{1} \mathrm{H}-\mathrm{NMR}\left(300 \mathrm{MHz}, \mathrm{DMSO}-d_{6}, \delta\right.$, ppm): 7.10 (1H, t, J: 4.84 Hz, Ar), $7.59(1 \mathrm{H}, \mathrm{ddd}, J: 7.63 \mathrm{~Hz}, 4.92 \mathrm{~Hz}$, $1.00 \mathrm{~Hz}, \mathrm{Ar}), 7.77(1 \mathrm{H}, \mathrm{s}, \mathrm{Ar}), 7.94-8.05(5 \mathrm{H}, \mathrm{m}, \mathrm{Ar}), 8.18(1 \mathrm{H}, \mathrm{d}, \mathrm{J}$ : $8.33 \mathrm{~Hz}, \mathrm{Ar}), 8.56(2 \mathrm{H}, \mathrm{d}, \mathrm{J}: 4.80 \mathrm{~Hz}, \mathrm{Ar}), 8.67(1 \mathrm{H}, \mathrm{d}, \mathrm{J}: 4.68 \mathrm{~Hz}, \mathrm{Ar})$, $10.88(1 \mathrm{H}, \mathrm{s}, \mathrm{NH}), 11.61(1 \mathrm{H}, \mathrm{s}, \mathrm{NH}) .{ }^{13} \mathrm{C}-\mathrm{NMR}\left(75 \mathrm{MHz}, \mathrm{DMSO}-d_{6}\right.$ ठ, ppm): 109.33, 116.22, 116.47, 121.35, 123.47, 129.81, 132.10, 138.49, 145.08, 149.32, 150.24, 151.79, 157.49, 158.82, 162.99. HR-MS (M+H): pred: 411.0692, found: 411.0690.

4-((4-(pyridine-3-yl)thiazol-2-yl)amino)-N-(pyrimidin-2yl)benzenesulfonamide (3e)

Yield: 68\%, m.p. $240.5^{\circ} \mathrm{C}$, IR v̄max $\left(\mathrm{cm}^{-1}\right)$ : 2988.66-2734.87 ( $\mathrm{Ar}$ C-H). ${ }^{~} \mathrm{H}-\mathrm{NMR}\left(300 \mathrm{MHz}, \mathrm{DMSO}-d_{6}, \delta, \mathrm{ppm}\right): 7.04(1 \mathrm{H}, \mathrm{t}, \mathrm{J}: 4.88$ $\mathrm{Hz}, \mathrm{Ar}), 7.59(1 \mathrm{H}, \mathrm{dd}, \mathrm{J}: 9.48 \mathrm{~Hz}, 4.92 \mathrm{~Hz}, \mathrm{Ar}), 7.72(1 \mathrm{H}, \mathrm{s}, \mathrm{Ar}), 7.87-$ 7.97 (4H, m, Ar), 8.42-8.51 (3H, m, Ar), 8.59 (1H, dd, J: 4.89 Hz, 1.45 $\mathrm{Hz}, \mathrm{Ar}), 9.22(1 \mathrm{H}, \mathrm{d}, \mathrm{J}: 1.74 \mathrm{~Hz}, \mathrm{Ar}), 10.85(1 \mathrm{H}, \mathrm{s}, \mathrm{NH}), 11.63(1 \mathrm{H}, \mathrm{s}$, $\mathrm{NH}) .{ }^{13} \mathrm{C}-\mathrm{NMR}\left(75 \mathrm{MHz}, \mathrm{DMSO}-d_{6}, \delta, \mathrm{ppm}\right): 163.2318,158.8155$, 157.4858, 147.5972, 147.2030, 146.1180, 145.0079, 135.0453, 132.1740, 130.9313, 129.8394, 124.8952, 116.5137, 116.2106, 107.3003. HR-MS (M+H): pred: 411.0692, found: 411.0692.

4-((4-(pyridine-4-yl)thiazol-2-yl)amino)-N-(pyrimidin-2yl)benzenesulfonamide (3f)

Yield: $80 \%$, m.p. $283^{\circ} \mathrm{C}$, IR ūmax $\left(\mathrm{cm}^{-1}\right)$ : $3609.64(\mathrm{~N}-\mathrm{H}), 2987.82-$ 2871.18 (Ar C-H). ${ }^{1} \mathrm{H}-\mathrm{NMR}$ (300 MHz, DMSO-d $\left.6, \delta, \mathrm{ppm}\right): 7.04$ $(1 \mathrm{H}, \mathrm{t}, \mathrm{J}: 4.88 \mathrm{~Hz}, \mathrm{Ar}), 7.84-8.01(7 \mathrm{H}, \mathrm{m}, \mathrm{Ar}), 8.50(2 \mathrm{H}, \mathrm{d}, \mathrm{J}: 4.86 \mathrm{~Hz}$, $\operatorname{Ar}), 8.62(2 \mathrm{H}, \mathrm{d}, \mathrm{J}: 5.76 \mathrm{~Hz}, \mathrm{Ar}), 10.85(1 \mathrm{H}, \mathrm{s}, \mathrm{NH}), 11.65(1 \mathrm{H}, \mathrm{s}, \mathrm{NH})$. ${ }^{13} \mathrm{C}-\mathrm{NMR}\left(75 \mathrm{MHz}\right.$, DMSO- $\left.d_{6}, \delta, \mathrm{ppm}\right): 109.51,116.22,116.52$, 120.54, 129.83, 132.21, 141.32, 144.98, 148.28, 150.62, 157.49, 158.82, 163.05. HR-MS (M+H): pred: 411.0692, found: 411.0694.

\section{$\mathrm{N}$-(pyridine-2-yl)-4-((4-(pyridine-2-yl)thiazol-2-yl)ami-} no)benzenesulfonamide ( $\mathbf{3 g})$

Yield: $75 \%$, m.p. $269^{\circ} \mathrm{C}$, IR v̄max $\left(\mathrm{cm}^{-1}\right): 3250.00(\mathrm{~N}-\mathrm{H}), 3112.13$ (N-H), 3010.80-2810.35 (Ar C-H). 'H-NMR (300 MHz, DMSO-d ${ }_{6}, \delta$, ppm): $6.81(1 \mathrm{H}, \mathrm{d}, \mathrm{J}: 4.60 \mathrm{~Hz}, \mathrm{Ar}), 7.24(1 \mathrm{H}, \mathrm{d}, \mathrm{J}: 4.66 \mathrm{~Hz}, \mathrm{Ar}), 7.33$ $(1 \mathrm{H}, \mathrm{ddd}, \mathrm{J}: 7.50 \mathrm{~Hz}, 4.80 \mathrm{~Hz}, 1.12 \mathrm{~Hz}, \mathrm{Ar}), 7.64(1 \mathrm{H}, \mathrm{s}, \mathrm{Ar}), 7.77-7.82$ (2H, m, Ar), 7.84-7.96 (5H, m, Ar), $8.07(1 \mathrm{H}, \mathrm{d}, \mathrm{J}$ : $7.89 \mathrm{~Hz}, \mathrm{Ar}), 8.59-$ ???? (1H, m, Ar), $10.73(1 \mathrm{H}, \mathrm{s}, \mathrm{NH}), 12.63(1 \mathrm{H}, \mathrm{s}, \mathrm{NH}) .{ }^{13} \mathrm{C}-\mathrm{NMR}(75$ MHz, DMSO- $\left.d_{6}, \delta, p p m\right): 108.42,116.67,121.08,123.25,124.84$ $127.86,134.54,137.81,144.46,149.87,150.89,152.38,163.02$, 169.05. HR-MS (M+H): pred: 410.0740, found: 410.0734.

\section{N-(pyridine-2-yl)-4-((4-(pyridine-3-yl)thiazol-2-yl)ami-} no)benzenesulfonamide (3h)

Yield: $64 \%$, m.p. $247.4^{\circ} \mathrm{C}$, IR v̄max $\left(\mathrm{cm}^{-1}\right)$ : $3241.06(\mathrm{~N}-\mathrm{H}), 3100$ 2785 (Ar C-H). ${ }^{1} \mathrm{H}-\mathrm{NMR}\left(300 \mathrm{MHz}, \mathrm{DMSO}-d_{6}, \delta, \mathrm{ppm}\right): 6.80(1 \mathrm{H}, \mathrm{s}$, Ar), $7.24(1 \mathrm{H}, \mathrm{br} . \mathrm{s}, \mathrm{Ar}), 7.46(1 \mathrm{H}, \mathrm{br} . \mathrm{s}, \mathrm{Ar}), 7.61(1 \mathrm{H}, \mathrm{br} . \mathrm{s}, \mathrm{Ar}), 7.82-$ $7.88(6 \mathrm{H}, \mathrm{m}, \mathrm{Ar}), 8.31(1 \mathrm{H}, \mathrm{m}, \mathrm{Ar}), 8.53(1 \mathrm{H}, \mathrm{br} . \mathrm{s}, \mathrm{Ar}), 9.18(1 \mathrm{H}, \mathrm{d}$ J: $2.01 \mathrm{~Hz}, \mathrm{Ar}), 10.77(1 \mathrm{H}, \mathrm{s}, \mathrm{NH}), 12.63(1 \mathrm{H}, \mathrm{s}, \mathrm{NH}) .{ }^{13} \mathrm{C}-\mathrm{NMR}(75$ MHz, DMSO- $d_{6} \delta$, ppm): 106.28, 108.43, 115.96, 116.74, 123.92, $124.29,124.84,125.21,127.11,127.90,128.85,130.46,133.58$, 134.61, 144.40, 144.64, 147.39, 147.75, 148.88, 163.27, 169.06. HR-MS (M+H): pred: 410.0740, found: 410.0736 .

\section{N-(pyridine-2-yl)-4-((4-(pyridine-4-yl)thiazol-2-yl)ami-} no)benzenesulfonamide (3i)

Yield: $70 \%$, m.p. $281^{\circ} \mathrm{C}$, IR v̄max $\left(\mathrm{cm}^{-1}\right)$ : $3251.75(\mathrm{~N}-\mathrm{H}), 3101.09$ (N-H), 2922.97-2779.28 (Ar C-H). ${ }^{1} \mathrm{H}-\mathrm{NMR}$ (300 MHz, DMSO-d 6 , ppm): $6.81(1 \mathrm{H}, \mathrm{d}, \mathrm{J}: 4.54 \mathrm{~Hz}, \mathrm{Ar}), 7.24(1 \mathrm{H}, \mathrm{d}, \mathrm{J}: 4.71 \mathrm{~Hz}, \mathrm{Ar}), 7.78-7.93$ $(9 \mathrm{H}, \mathrm{m}, \mathrm{Ar}), 8.63(2 \mathrm{H}, \mathrm{d}, \mathrm{J}: 5.37 \mathrm{~Hz}, \mathrm{Ar}), 10.79(1 \mathrm{H}, \mathrm{s}, \mathrm{NH}), 12.63(1 \mathrm{H}, \mathrm{s}$, $\mathrm{NH}) .{ }^{13} \mathrm{C}-\mathrm{NMR}$ (75 MHz, DMSO-d 6 , $\delta$, ppm): 108.44, 109.38, 116.77, 120.57, 124.85, 127.86, 134.72, 141.49, 144.29, 148.22, 150.48, 163.17, 169.06. HR-MS (M+H): pred: 410.0740, found: 410.0737.

N-(5-methylisoxazol-3-yl)-4-((4-(pyridine-2-yl)thiazol2-yl)amino)benzenesulfonamide (3j)

Yield: 85\%, m.p. $235^{\circ} \mathrm{C}$, IR v̄max $\left(\mathrm{cm}^{-1}\right): 3360.37(\mathrm{~N}-\mathrm{H}), 3223.83(\mathrm{~N}-\mathrm{H})$, 3098.93-3005.34 (Ar C-H). ${ }^{1} \mathrm{H}-\mathrm{NMR}$ (300 MHz, DMSO-d $\left.d_{6}, \delta, p p m\right)$ : $2.29(3 \mathrm{H}, \mathrm{s}, \mathrm{Ar}), 6.13(1 \mathrm{H}, \mathrm{s}, \mathrm{Ar}), 7.59(1 \mathrm{H}, \mathrm{t}, \mathrm{J}: 6.18 \mathrm{~Hz}, \mathrm{Ar}), 7.84(2 \mathrm{H}, \mathrm{d}$, J: $8.97 \mathrm{~Hz}, \mathrm{Ar}), 7.94-7.98(3 \mathrm{H}, \mathrm{m}, \mathrm{Ar}), 8.19(1 \mathrm{H}, \mathrm{t}, \mathrm{J}: 8.26 \mathrm{~Hz}, \mathrm{Ar}), 8.69$ $(1 \mathrm{H}, \mathrm{d}, \mathrm{J}: 5.16 \mathrm{~Hz}, \mathrm{Ar}), 10.98(1 \mathrm{H}, \mathrm{s}, \mathrm{NH}), 11.30(1 \mathrm{H}, \mathrm{s}, \mathrm{NH}) .{ }^{13} \mathrm{C}-\mathrm{NMR}$ (75 MHz, DMSO-d $d_{6} \delta$, ppm): 12.53, 95.83, 112.20, 117.14, 122.44, 124.29, 128.95, 130.89, 131.44, 141.43, 145.13, 146.94, 158.10, 163.34, 170.67. HR-MS (M+H): pred: 414.0689, found: 414.0695.

\section{N-(5-methylisoxazol-3-yl)-4-((4-(pyridine-3-yl)thiazol-} 2-yl)amino)benzenesulfonamide (3k)

Yield: $80 \%$, m.p. $254.5^{\circ} \mathrm{C}$, IR v̄max $\left(\mathrm{cm}^{-1}\right): 3439.15(\mathrm{~N}-\mathrm{H}), 3339.96$ (NH), 3162.61-2875.16 (Ar C-H). ${ }^{1} \mathrm{H}-\mathrm{NMR}\left(300 \mathrm{MHz}, \mathrm{DMSO}_{-}{ }_{6}, \delta_{\text {, }}\right.$ ppm): $2.29(3 \mathrm{H}, \mathrm{s}, \mathrm{Ar}), 6.14(1 \mathrm{H}, \mathrm{s}, \mathrm{Ar}), 7.47(1 \mathrm{H}, \mathrm{dd}, \mathrm{J}: 6.09 \mathrm{~Hz}, 4.8 \mathrm{~Hz}$, Ar), 7.66(1H, s, Ar), 7.82-7.93 (4H, m, Ar), $8.31(1 \mathrm{H}, \mathrm{dt}, \mathrm{J}: 8.05 \mathrm{~Hz}, 1.9$ $\mathrm{Hz}, \mathrm{Ar}), 8.53(1 \mathrm{H}, \mathrm{dd}, \mathrm{J}: 4.56 \mathrm{~Hz}, 1.55 \mathrm{~Hz}, \mathrm{Ar}), 9.17(1 \mathrm{H}, \mathrm{d}, \mathrm{J}: 2.01 \mathrm{~Hz}$, Ar), $10.88(1 \mathrm{H}, \mathrm{s}, \mathrm{NH}), 11.28(1 \mathrm{H}, \mathrm{s}, \mathrm{NH}) .{ }^{13} \mathrm{C}-\mathrm{NMR}(75 \mathrm{MHz}, \mathrm{DMSO}$ $\left.d_{6}, \delta, p p m\right): 170.6549,163.0586,158.1270,149.0320,147.8212$ 147.4862, 145.3790, 133.5084, 131.1830, 130.3561, 129.0094, 124.2756, 116.9107, 106.6712, 95.8413, 95.8156, 12.5345 HR-MS $(\mathrm{M}+\mathrm{H})$ : pred: 414.0689, found: 414.0697

\section{N-(5-methylisoxazol-3-yl)-4-((4-(pyridine-4-yl)thiazol-} 2-yl)amino)benzenesulfonamide (3I)

Yield: 65\%, m.p. $290.5^{\circ} \mathrm{C}$, IR v̄max ( $\left.\mathrm{cm}^{-1}\right)$ : $3320.74(\mathrm{~N}-\mathrm{H}), 3185.88$ (N-H), 3114.95-2923.28 (Ar C-H). ${ }^{1} \mathrm{H}-\mathrm{NMR}\left(300 \mathrm{MHz}, \mathrm{DMSO}^{-} d_{6}, \delta\right.$ ppm): 2.29 (3H, s, Ar), 6.14 (1H, s, Ar), 7.85-7.91 (7H, m, Ar), 8.63 $(2 \mathrm{H}, \mathrm{d}, \mathrm{J}: 6.09 \mathrm{~Hz}, \mathrm{Ar}), 10.90(1 \mathrm{H}, \mathrm{s}, \mathrm{NH}), 11.29(1 \mathrm{H}, \mathrm{s}, \mathrm{NH}) .{ }^{13} \mathrm{C}-\mathrm{NMR}$ 
(75 MHz, DMSO- $d_{6}, \delta$, ppm): 12.54, 95.82, 109.60, 116.95, 120.52, 129.00, 131.29, 141.25, 145.30, 148.31, 150.68, 158.12, 162.99. HR-MS (M+H): pred: 414.0689, found: 414.0695.

\section{4-((4-(pyridine-2-yl)thiazol-2-yl)amino)-N-(thiazol-2-yl) benzenesulfonamide (3m)}

Yield: $72 \%$, m.p. $266.8^{\circ} \mathrm{C}$, IR v̄max $\left(\mathrm{cm}^{-1}\right)$ : $3279.64(\mathrm{~N}-\mathrm{H}), 3149.35-$ 2811.30 (Ar C-H). ${ }^{1} \mathrm{H}-\mathrm{NMR}$ (300 MHz, DMSO-d $\left., \delta, \mathrm{ppm}\right): 6.81$ (1H, d, J: $4.62 \mathrm{~Hz}$, thiazole), $7.24(1 \mathrm{H}, \mathrm{d}, \mathrm{J}: 4.62 \mathrm{~Hz}$, thiazole), 7.3304-7.38 (1H, m, Ar), $7.66(1 \mathrm{H}, \mathrm{s}, \mathrm{Ar}), 7.68-7.80(2 \mathrm{H}, \mathrm{m}, \mathrm{Ar})$, 7.85-7.95 (3H, m, Ar), $8.09(1 \mathrm{H}, \mathrm{d}, \mathrm{J}: 7.89 \mathrm{~Hz}, \mathrm{Ar}), 8.59-8.61(1 \mathrm{H}, \mathrm{m}$, Ar) $10.74(1 \mathrm{H}, \mathrm{s}, \mathrm{NH}), 12.64(1 \mathrm{H}, \mathrm{s}, \mathrm{NH}) .{ }^{13} \mathrm{C}-\mathrm{NMR}(75 \mathrm{MHz}, \mathrm{DM}-$ SO- $d_{6}, \delta$, ppm): 108.42, 108.73, 116.68, 121.20, 123.34, 124.82, 127.84, 134.56, 138.12, 144.42, 149.61, 150.57, 152.13, 163.05. HR-MS (M+H): pred: 416.0304, found: 416.0297.

\section{4-((4-(pyridine-3-yl)thiazol-2-yl)amino)-N-(thiazol-2-yl) benzenesulfonamide (3n)}

Yield: 75\%, m.p. $292^{\circ} \mathrm{C}$, IR (v̄max $\left(\mathrm{cm}^{-1}\right)$ : $3217.14(\mathrm{~N}-\mathrm{H}), 3165.09$ (N-H), 3029-2998 (Ar C-H). ${ }^{1} \mathrm{H}-\mathrm{NMR}\left(300 \mathrm{MHz}, \mathrm{DMSO}-d_{6}, \delta, \mathrm{ppm}\right)$ : $6.82(1 \mathrm{H}, \mathrm{d}, \mathrm{J}: 4.59 \mathrm{~Hz}$, thiazole), $7.25(1 \mathrm{H}, \mathrm{d}, \mathrm{J}: 4.62 \mathrm{~Hz}$, thiazole), $7.79(2 \mathrm{H}, \mathrm{d}, \mathrm{J}: 8.85 \mathrm{~Hz}, \mathrm{Ar}), 7.91(2 \mathrm{H}, \mathrm{d}, \mathrm{J}: 8.82 \mathrm{~Hz}, \mathrm{Ar}), 7.98(1 \mathrm{H}$, s, thiazole), $8.05(1 \mathrm{H}, \mathrm{dd}, \mathrm{J}: 9 \mathrm{~Hz}, 5.60 \mathrm{~Hz}, \mathrm{Ar}), 8.82(1 \mathrm{H}, \mathrm{d}, \mathrm{J}: 5.43$ $\mathrm{Hz}, \mathrm{Ar}), 8.99(1 \mathrm{H}, \mathrm{d}, \mathrm{J}: 8.37 \mathrm{~Hz}, \mathrm{Ar}), 9.40(1 \mathrm{H}, \mathrm{s}, \mathrm{NH}), 10.95(1 \mathrm{H}, \mathrm{s}$, $\mathrm{NH}) .{ }^{13} \mathrm{C}-\mathrm{NMR}\left(75 \mathrm{MHz}, \mathrm{DMSO}-d_{6}, \delta, \mathrm{ppm}\right): 169.0689,163.7379$, $144.7641,144.0954,141.8711,141.1882,140.6548,134.9036$, 133.1460, 127.8172, 127.3908, 124.8855, 116.9819, 110.1459, 108.4805. HR-MS (M+H): pred: 416.0304, found: 416.0302.

\section{4-((4-(pyridine-4-yl)thiazol-2-yl)amino)-N-(thiazol-2-yl) benzenesulfonamide (30)}

Yield: 90\%, m.p. Decomposed, IR v̄max $\left(\mathrm{cm}^{-1}\right)$ : $3212.97(\mathrm{~N}-\mathrm{H})$, $3157.92(\mathrm{~N}-\mathrm{H}), 3091.73-2780.85$ (Ar C-H). ' $\mathrm{H}-\mathrm{NMR}(300 \mathrm{MHz}$, DMSO- $\left.d_{6}, \delta, p p m\right): 6.83(1 \mathrm{H}, \mathrm{d}, J: 4.62 \mathrm{~Hz}$, thiazole), $7.25(1 \mathrm{H}, \mathrm{d}$, J: $4.62 \mathrm{~Hz}$, thiazole), $7.79(2 \mathrm{H}, \mathrm{d}, \mathrm{J}: 8.82 \mathrm{~Hz}, \mathrm{Ar}), 7.91(2 \mathrm{H}, \mathrm{d}, \mathrm{J}: 8.79$ $\mathrm{Hz}, \mathrm{Ar}), 8.42(1 \mathrm{H}, \mathrm{s}$, thiazole), $8.51(2 \mathrm{H}, \mathrm{d}, \mathrm{J}: 6.45 \mathrm{~Hz}, \mathrm{Ar}), 8.91(2 \mathrm{H}$, d, J: $6.63 \mathrm{~Hz}, \mathrm{Ar}), 11.05(1 \mathrm{H}, \mathrm{s}, \mathrm{NH}), 12.67(1 \mathrm{H}, \mathrm{s}, \mathrm{NH}) .{ }^{13} \mathrm{C}-\mathrm{NMR}$ (75 MHz, DMSO- $d_{6} \delta$, ppm): 169.0807, 163.6579, 148.8425, 145.8981, 143.9361, 142.9127, 135.1140, 127.8246, 124.8885, 122.8679, 117.0760, 108.5102. HR-MS (M+H): pred: 416.0304, found: 416.0299 .

\section{4-((4-(pyridine-2-yl)thiazol-2-yl)amino)benzenesulfon- amide (3p)}

Yield: 60\%, m.p. $242^{\circ} \mathrm{C}$, IR v̄max $\left(\mathrm{cm}^{-1}\right)$ : 3340.25, $3269.36(\mathrm{~N}-\mathrm{H})$, 3190.39 (N-H), 3123.55-3007.22 (Ar C-H). ' H-NMR (300 MHz, DMSO- $\left.d_{6}, \delta, p p m\right): 7.23\left(2 \mathrm{H}, \mathrm{s}, \mathrm{SO}_{2} \mathrm{NH}_{2}\right), 7.31-7.36(1 \mathrm{H}, \mathrm{m}, \mathrm{Ar})$, $7.65(1 \mathrm{H}, \mathrm{s}$, thiazole), 7.79-7.94 $(5 \mathrm{H}, \mathrm{m}, \mathrm{Ar}), 8.07(1 \mathrm{H}, \mathrm{d}, \mathrm{J}: 7.86 \mathrm{~Hz}$, Ar), 8.59-8.61 (1H, m, Ar), $10.74(1 \mathrm{H}, \mathrm{s}, \mathrm{NH}) .{ }^{13} \mathrm{C}-\mathrm{NMR}(75 \mathrm{MHz}$, DMSO- $d_{6}, \delta$, ppm): 163.1068, 152.3861, 150.8621, 149.9034, 144.2033, 137.8423, 136.5434, 127.6186, 123.2630, 121.0212, 116.6925, 108.4336. HR-MS $(M+H)$ : pred: 333.0474, found: 333.0470 .

\section{4-((4-(pyridine-3-yl)thiazol-2-yl)amino)benzenesulfon- amide (3q)}

Yield: 70\%, m.p. $290^{\circ} \mathrm{C}$, IR v̄max $\left(\mathrm{cm}^{-1}\right)$ : 3380.7, $3309.18(\mathrm{~N}-\mathrm{H})$, $3225.50(\mathrm{~N}-\mathrm{H}), 3126.74-2814.21$ (Ar C-H). ${ }^{1} \mathrm{H}-\mathrm{NMR}(300 \mathrm{MHz}$, DMSO- $\left.d_{6}, \delta, p p m\right): 7.24\left(2 \mathrm{H}, \mathrm{s}, \mathrm{SO}_{2} \mathrm{NH}_{2}\right), 7.48(1 \mathrm{H}, \mathrm{dd}, \mathrm{J}: 8.01 \mathrm{~Hz}$, $4.79 \mathrm{~Hz}, \mathrm{Ar}), 7.64(1 \mathrm{H}, \mathrm{s}$, thiazole), 7.79-7.87 $(4 \mathrm{H}, \mathrm{m}, \mathrm{Ar}), 8.11(1 \mathrm{H}$, dt, J: $8.1 \mathrm{~Hz}, 1.95 \mathrm{~Hz}, \mathrm{Ar}), 8.52-8.54(1 \mathrm{H}, \mathrm{m}, \mathrm{Ar}), 9.17-$ ????? (1H, $\mathrm{m}, \mathrm{Ar}), 10.75(1 \mathrm{H}, \mathrm{s}, \mathrm{NH}) .{ }^{13} \mathrm{C}-\mathrm{NMR}\left(75 \mathrm{MHz}, \mathrm{DMSO}-d_{6}, \delta, \mathrm{ppm}\right)$ : 106.24, 116.75, 124.29, 127.63, 130.44, 133.45, 136.63, 144.13, 147.47, 147.77, 149.00, 163.34. HR-MS (M+H): pred: 333.0474, found: 333.0466 .

\section{4-((4-(pyridine-4-yl)thiazol-2-yl)amino)benzenesulfon- amide (3r)}

Yield: $80 \%$, m.p. $249.5^{\circ} \mathrm{C}$, IR v̄max $\left(\mathrm{cm}^{-1}\right)$ : 3332.95, 3299.27 (N$\mathrm{H}), 3231.70(\mathrm{NH}), 3174.5-3007.22$ (Ar C-H). ${ }^{1} \mathrm{H}-\mathrm{NMR}(300 \mathrm{MHz}$, DMSO- $\left.d_{6}, \delta, p p m\right): 7.23\left(2 \mathrm{H}, \mathrm{s}_{1} \mathrm{SO}_{2} \mathrm{NH}_{2}\right), 7.80-7.91(4 \mathrm{H}, \mathrm{m}, \mathrm{Ar})$, $8.03(1 \mathrm{H}, \mathrm{s}$, thiazole), $8.11(2 \mathrm{H}, \mathrm{d}, \mathrm{J}: 6.09 \mathrm{~Hz}, \mathrm{Ar}), 8.73(2 \mathrm{H}, \mathrm{d}, \mathrm{J}$ : $5.60 \mathrm{~Hz}$, py 2,6-H), $10.87(1 \mathrm{H}, \mathrm{s}, \mathrm{NH}) .{ }^{13} \mathrm{C}-\mathrm{NMR}\left(75 \mathrm{MHz}, \mathrm{DMSO}-d_{6}\right.$, ठ, ppm): 111.91, 116.91, 121.32, 127.62, 136.87, 143.93, 144.00, 147.42, 147.94 HR-MS (M+H): pred: 333.0474, found: 333.0478.

\section{Antimicrobial activity \\ Minimum inhibitory concentration assay}

Antimicrobial activity test was applied to various gram positive and gram negative bacteria strains including, E. coli (ATCC8739), S. aureus (ATCC6538), B. spizizenii (ATCC6633), K. pneumoniae (Clinical isolate), P.aeruginosa (ATCC9027), E. faecalis(ATCC29212), S. epidermidis (ATCC12228), VRE (Clinical isolate). These organisms were inoculated to mid-log phase in Muller Hinton Broth $(\mathrm{MHB})$ at $37^{\circ} \mathrm{C}$. Broth microdilution procedure is a more userfriendly method that enables testing of multiple antimicrobial agents. The broth microdilution method was carried out in accordance with the relevant 2018 CLSI standard. Compounds were dissolved in DMSO below 1\% concentration and were added to 96-well plates. These compounds were 2-fold serially diluted to make different concentrations, from 0.5 to $256 \mathrm{mM}$. Bacterial inoculum suspensions were prepared at a final concentration of $1 \times 10^{5} \mathrm{cfu} / \mathrm{ml}$ and plates were incubated at $37^{\circ} \mathrm{C}$ for 24 hours. Positive or negative controls were set to wells with and without bacteria, respectively. Sulfamethoxazole and chloramphenicol were used as standards. The MIC was determined by visual inspection after the change in turbidity. Experiments were performed in triplicate.

\section{RESULTS AND DISCUSSION}

\section{Chemistry}

Tested compounds were synthesized successfully by the proposed methods in $60-85 \%$ yield. $\mathrm{NH}$ stretchings were observed above $3.000 \mathrm{~cm}^{-1}$ and $\mathrm{C}-\mathrm{H}$ stretchings were observed between $2.800-3.100 \mathrm{~cm}^{-1}$ in the IR spectra. All protons matched with the expectations in ${ }^{1} \mathrm{H}-\mathrm{NMR}$. NH protons for $\mathbf{3 a - 0}$ were observed as two separated singlet peaks around $\delta \sim 10.8$ and $\sim 11.55$ ppm. Supporting the literature information, $\mathrm{SO}_{2} \mathrm{NH}_{2}$ containing (3pr) molecules $\mathrm{NH}$ stretchings were observed at $\mathrm{d} 7.23 \mathrm{ppm}$ and the other $\mathrm{NH}$ group stretchings observed at $\delta \sim 10.8 \mathrm{ppm}$ like the rest in the series (Gowda, Jyothi, \& D'Souza, 2002; Başar, Tunca, Bülbül, \& Kaya, 2016). The hydrogen on the neighborhood of the pyridine nitrogen was observed approximately $d$ 8-9 ppm for pyridine/pyrimidine containing molecules as singlet or mixed by aromatic hydrogens as multiplet. Most of the single aromatic hydrogens at thiazole and/or isoxazole rings were observed around d 8.2 and 6.2 ppm, respectively. Some of them were interfered by other aromatic peaks and observed as multiplet. Methyl groups on $\mathbf{1 - 3}$ and $\mathbf{1 0 - 1 2}$ were observed 
around $\delta \sim 2.25 \mathrm{ppm}$. In the ${ }^{13} \mathrm{C}$ NMR, methyl-containing compounds gave a $\delta \sim 20$ ppm peak. All other carbons were aromatics, thus were observed between d 120-160 ppm. Finally, HRMS results proved the structures of the molecules.

\section{Antimicrobial activity}

Antimicrobial activity of the tested compounds are given in Table 1. Among the series, 6 of the 18 compounds exhibited lower MIC values than $256 \mathrm{\mu g} / \mathrm{mL}$. The MIC value of $\mathbf{3 d}$, sulfadiazine-thiazole 2-pyridyle derivative was $64 \mu \mathrm{g} / \mathrm{mL}$ against S. aureus. The other sulfadiazine derivatives $(\mathbf{3 e}, \mathbf{3 f})$ were also found more active compared to the rest of the series. 3-pyridyle derivative (3e), exhibited $128 \mu \mathrm{g} / \mathrm{mL}$ against S. aureus and E. faecalis. Compound $3 e$ also showed $32 \mu \mathrm{g} / \mathrm{mL}$ against S.epidermidis. 4-pyridyle derivative of sulfadiazine (3f) exhibited $64 \mu \mathrm{g} / \mathrm{mL}$ against S.epidermidis. Sulfanilamide derivatives (3p-r) exhibited 32 and $128 \mu \mathrm{g} / \mathrm{mL}$ against B.spizizenii and S.aureus, respectively. Besides, 3q exhibited $64 \mu \mathrm{g} / \mathrm{ml}$ against VRE, as the only effective compound against this microorganism. $\mathbf{3 r}$ exhibited $128 \mu \mathrm{g} / \mathrm{mL}$ against E.coli and P.aeruginosa as the only compound that showed inhibitory activity against gram-negative species along with $\mathbf{3 q}$. The standard drug sulfamethoxazole showed 4, 32 and $64 \mu \mathrm{g} / \mathrm{mL}$ against E.coli, P.aeruginosa and S.aureus, respectively. The other standard drug chloramphenicol showed 8-16 $\mu \mathrm{g} / \mathrm{mL}$ against all micro- organisms as a wide spectrum antibiotic. None of the compounds showed inhibitory effect against K.pneumoniae, and there was only one compound (3r), which showed inhibition against gram-negative bacteria growth. The compounds were derived from two main parts; those which are sulfonamide derivatives and the position of pyridyl substitution. To point this out, 6 compounds seemed to be more effective compared to the rest of the series, and these 6 compounds were sulfanilamide and sulfadiazine derivatives. There was no significant data difference between 2, 3 or 4-pyridyl derivatives of each sulfonamide derivative. Considering these results, it was interpreted that activity was mainly related to the sulfonamide part. Besides, the pyridyl position did not make an important change in inhibition. High inhibitor potential $(32 \mu \mathrm{g} / \mathrm{mL})$ was provided by compound 3p-r, which supports the SAR feature that an unsubstituted amine group is important for activity in sulfonamide derivatives.

Acknowledgements: Authors declare that there is no conflict of interest for this work.

Peer-review: Externally peer-reviewed.

Author Contributions: Conception/Design of Study- Z.Ş., Ş.D.; Data Acquisition- L.Y., Z.Ş., S.N.B.; Data Analysis/Interpretation- Z.S.., S.N.B. Drafting Manuscript- Z.S., S.N.B., L.Y.; Critical Revision of Manuscript-

Table 1. Screening for MIC of the compounds 3a-r using microdilution method.

\begin{tabular}{|c|c|c|c|c|c|c|c|c|}
\hline \multirow{3}{*}{ Cpd. } & \multicolumn{8}{|c|}{ Microorganisms and minimal inhibitory concentration $(\mu \mathrm{g} / \mathrm{mL})$} \\
\hline & \multicolumn{3}{|c|}{ Gram-Negative Bacteria } & \multicolumn{5}{|c|}{ Gram-Positive Bacteria } \\
\hline & E.coli & P.aeruginosa & K.pneumoniae & B.spizizenii & S.aureus & E.faecalis & S.epidermidis & VRE \\
\hline $3 a$ & $>256$ & $>256$ & $>256$ & $>256$ & $>256$ & $>256$ & $>256$ & $>256$ \\
\hline $3 \mathbf{b}$ & $>256$ & $>256$ & $>256$ & $>256$ & $>256$ & $>256$ & $>256$ & $>256$ \\
\hline $3 c$ & $>256$ & $>256$ & $>256$ & $>256$ & $>256$ & $>256$ & $>256$ & $>256$ \\
\hline $3 d$ & $>256$ & $>256$ & $>256$ & $>256$ & $>64$ & $>256$ & $>128$ & $>256$ \\
\hline $3 e$ & $>256$ & $>256$ & $>256$ & $>256$ & $>128$ & $>128$ & $>32$ & $>256$ \\
\hline $3 f$ & $>256$ & $>256$ & $>256$ & $>256$ & $>256$ & $>256$ & $>64$ & $>256$ \\
\hline $3 g$ & $>256$ & $>256$ & $>256$ & $>256$ & $>256$ & $>256$ & $>256$ & $>256$ \\
\hline $3 \mathrm{~h}$ & $>256$ & $>256$ & $>256$ & $>256$ & $>256$ & $>256$ & $>256$ & $>256$ \\
\hline $3 i$ & $>256$ & $>256$ & $>256$ & $>256$ & $>256$ & $>256$ & $>256$ & $>256$ \\
\hline $3 j$ & $>256$ & $>256$ & $>256$ & $>256$ & $>256$ & $>256$ & $>256$ & $>256$ \\
\hline $3 k$ & $>256$ & $>256$ & $>256$ & $>256$ & $>256$ & $>256$ & $>256$ & $>256$ \\
\hline 31 & $>256$ & $>256$ & $>256$ & $>256$ & $>256$ & $>256$ & $>256$ & $>256$ \\
\hline $3 m$ & $>256$ & $>256$ & $>256$ & $>256$ & $>256$ & $>256$ & $>256$ & $>256$ \\
\hline $3 n$ & $>256$ & $>256$ & $>256$ & $>256$ & $>256$ & $>256$ & $>256$ & $>256$ \\
\hline 30 & $>256$ & $>256$ & $>256$ & $>256$ & $>256$ & $>256$ & $>256$ & $>256$ \\
\hline $3 p$ & $>256$ & $>256$ & $>256$ & $>32$ & $>128$ & $>256$ & $>256$ & $>256$ \\
\hline $3 q$ & $>256$ & $>256$ & $>256$ & $>32$ & $>128$ & $>256$ & $>256$ & $>64$ \\
\hline $3 r$ & $>128$ & $>128$ & $>256$ & $>32$ & $>128$ & $>256$ & $>256$ & $>128$ \\
\hline C & $>8$ & $>16$ & $>8$ & $>16$ & $>16$ & $>8$ & $>8$ & $>16$ \\
\hline$S$ & $>4$ & $>32$ & $>256$ & $>256$ & $>64$ & $>256$ & $>256$ & $>256$ \\
\hline
\end{tabular}


Z.Ş., Ş.D.; Final Approval and Accountability- Z.Ş., S.N.B., L.Y., Ş.D.; Technical or Material Support- L.Y., Z.Ş., S.N.B.; Supervision- Ş.D., L.Y.

Conflict of Interest: The authors have no conflict of interest to declare.

Financial Disclosure: Authors declared no financial support.

\section{REFERENCES}

- Başar, E., Tunca, E., Bülbül, M., \& Kaya, M. (2016). Synthesis of novel sulfonamides under mild conditions with effective inhibitory activity against the carbonic anhydrase isoforms I and II. Journal of Enzyme Inhibition and Medicinal Chemistry, 31(6), 1356-1361.

- Bengoechea, J. A., \& Pessoa, J. S. (2018). Klebsiella pneumoniae infection biology: living to counteract host defences. FEMS Microbiology Reviews, 43(2), 123-144.

- Donnenberg, M. S., \&Whittam, T. S. (2001). Pathogenesis and evolution of virulence in enteropathogenic and enterohemorrhagic Escherichia coli. Journal of Clinical Investigation, 107(5), 539-548.

- Drobniewski, F. A. (1993). Bacillus cereus and related species. Clinical Microbiology Reviews, 6(4), 324-338.

- $\quad$ El-Sayed, A., Solimana, M., El-Zahar, I., El-Masry, A. H., Kamel, M., \& Gohar, R. S. (2010). Synthesis and anticancer evaluation of novel tetrahydronaphthalen-6-yl thiazole heterocycles against human HePG2 and MCF7 cell lines. Der Pharma Chemica 2(5), 507-521.
Ertas, M., Sahin, Z., Berk, B., Yurttas, L., Biltekin, S. N., \& Demirayak, S. (2018). Pyridine-substituted thiazolylphenol derivatives: Synthesis, modeling studies, aromatase inhibition, and antiproliferative activity evaluation. Archiv der Pharmazie, 351(3-4), 1700272.

- Gellatly, S. L., \& Hancock, R. E.W. (2013). Pseudomonas aeruginosa: new insights into pathogenesis and host defenses. Pathogens and Disease, 67(3), 159-173.

- Gowda, B. T., Jyothi, K., \& D'Souza, J. D. (2002). Infrared and NMR spectra of arylsulphonamides. Zeitschrift für Naturforschung A, 57a, 967-973.

- Hamada, Y. (2018). Role of Pyridines in Medicinal Chemistry and Design of BACE1 Inhibitors Possessing a Pyridine Scaffold. Pyridine, doi:10.5772/intechopen.74719.

- Kashyap, S. J., Garg, V. K., Sharma, P. K., Kumar, N., Dudhe, R., \& Gupta, J. K. (2011). Thiazoles: having diverse biological activities. Medicinal Chemistry Research, 21(8), 2123-2132.

- $\quad$ Russo, T. A., \& Marr, C. M. (2019). Hypervirulent Klebsiella pneumoniae. Clinical Microbiology Reviews, doi:10.1128/cmr.00001-19.

- Sun, S., Jia, Q., Zenova, A. Y., Chafeev, M., Zhang, Z., Lin, S., \& Oballa, R. M. (2014). The discovery of benzenesulfonamide-based potent and selective inhibitors of voltage-gated sodium channel $\mathrm{Na} \mathrm{v}$ 1.7. Bioorganic \& Medicinal Chemistry Letters, 24(18), 4397-4401.

- Supuran, C. (2017). Special Issue: Sulfonamides. Molecules, 22(10), 1642. doi:10.3390/molecules22101642. 